\title{
Zur Misopädie von Urbanisierung und schulischer Bildung: Eine Würdigung Colin Wards
}

,Das Kind in der Stadt'

Kommentare von:

Imbke Behnken, Anika Duveneck, Tanu Biswas

Kommentar zu Colin Ward „Das Kind in der Stadt (Auszüge)“ (2021 [1978])

Was heißt es, Colin Ward einen Akt des Denkens (als Schreiben) zu widmen? Für mich bedeutet es, innezuhalten, um die Misopädie der modernen Urbanisierung und des damit verbundenen Schulsystems zu erkennen und aufmerksam zu studieren. Der Ausdruck Misopädie ist verwandt mit den Begriffen Misogynie und Misanthropie. Er bezeichnet eine Antipathie gegenüber Kindern und Kindheit (vgl. Rollo 2018) und in der wissenschaftlichen und gesellschaftlichen Auseinandersetzung bleibt ein großer blinder Fleck. Es geht, anders gesagt, um Adultismus (vgl. Bonnardel 2015; Liebel 2020). Sich mit einem Denker wie Colin Ward zu befassen bedeutet, sich ernsthaft zu bemühen, Kinder und Kindheit lieben zu lernen, indem man erkennt, dass die Moderne Kinder und Kindheit systematischer Unfreiheit, Marginalisierung und Unterdrückung aussetzt.

Städte stehen Kindern und Kindheit ausgesprochen gleichgültig gegenüber, wie Ward an diversen Beispielen zeigt. Ich gehe davon aus, dass Misopädie ein Grundpfeiler neuzeitlicher industrieller Bestrebungen ist, die in Urbanisierung und Beschulung ihren maßgeblichen Ausdruck finden. Der Stadtplanung und der schulischen Bildung ging es nie um Fragen der intergenerationellen Nachhaltigkeit oder der Herausbildung von Gemeinschaft. Erst seit Kurzem beginnen einige Theoretiker*innen und Praktiker*innen zu fragen: „In welcher Art städtischer Räume wollen wir Kinder aufwachsen sehen?“ (Vgl. Gill 2021) Geht man von einer derartigen Fragestellung aus, ist es unwahrscheinlich, dass am Ende autodominierte Masterpläne herauskommen oder Stadtlandschaften mit speziellen „Orten“ für Kinder, die diese nur unter Aufsicht von Erwachsenen erreichen können. Junge Klimaaktivist*innen kritisieren ebenfalls auf vielfältige Weise die Misopädie der industriellen Urbanisierung, obwohl sie den Begriff selbst nicht verwenden. Politische Plakate klimaengagierter Kindergartenkinder, die ich 2019 während des globalen Streiks für Klimagerechtigkeit in Berlin getroffen habe, scheinen mir eine ähnliche Botschaft zu senden.

Die 15-jährige Raina Ivanova aus Hamburg findet, dass sich die Erfahrung, zur Schule zu gehen, geändert hat. Raina ist eine der 16 Kläger*innen, die im Rahmen der UN-Kinderrechtskonvention gemeinsam fünf Staaten 


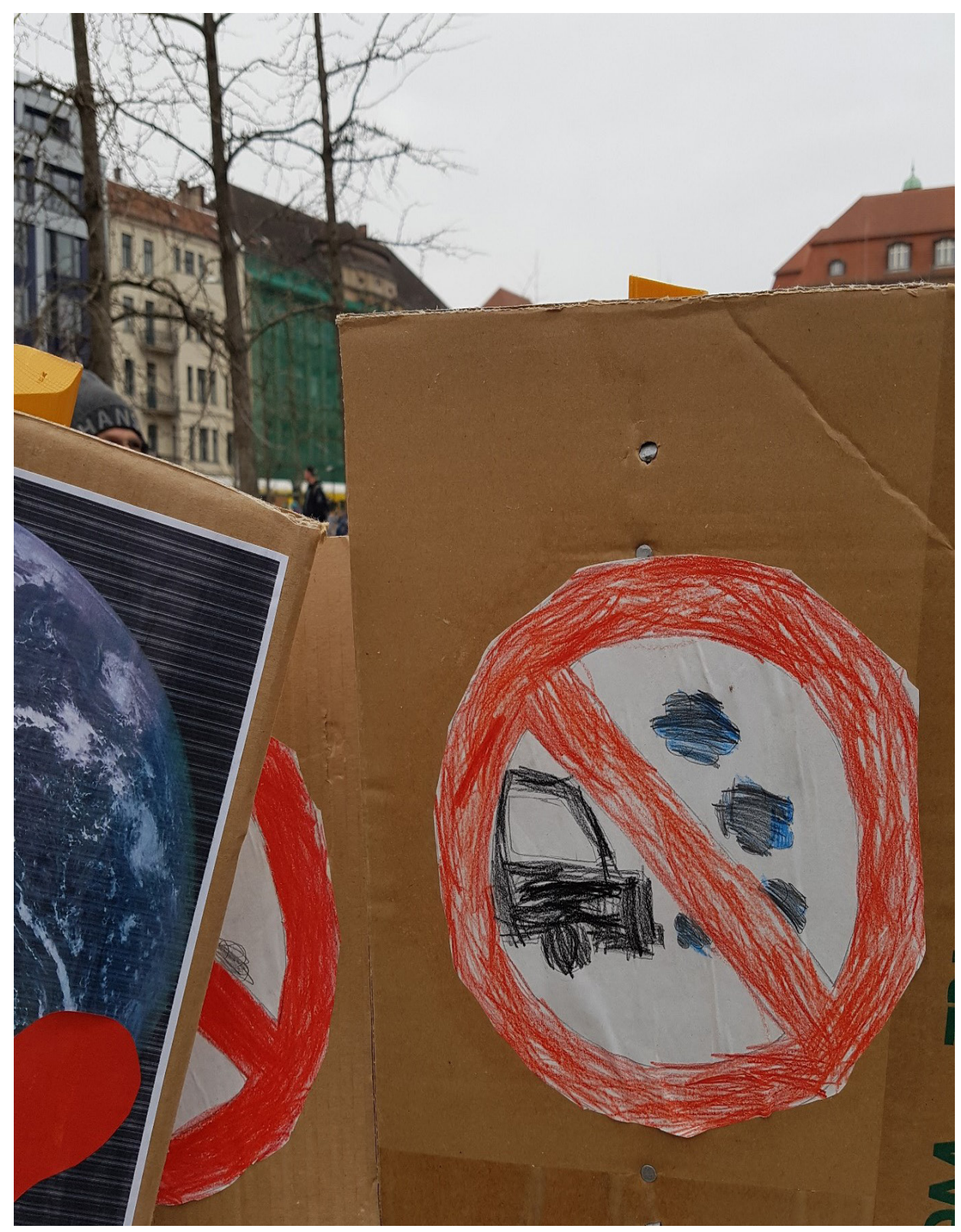

- nämlich Argentinien, Brasilien, Frankreich, Deutschland und die Türkei - wegen Verletzung ihrer Rechte verklagt haben (vgl. Earth Justice 2019). Hitzewellen und extreme Temperaturen im Frühling und Sommer zwingen Raina und ihre Klassenkamerad*innen, den Unterricht zu unterbrechen und ihre Klassenräume zu wechseln, weil sie sich in der stickig-schwülen Luft schlechter aufs Lernen konzentrieren können. Die Kanäle in ihrer Stadt haben einen zu niedrigen Wasserstand, was den Bootsverkehr erschwert. Vor allem 2017 erlebte Hamburg Starkregenfälle, schwere Stürme und Überschwemmungen, die das Eigentum von Rainas Freund*innen und Nachbar*innen zerstörten. Auf dem Fußballplatz ihrer Schule wateten Raina und ihre Freund*innen durch knietiefes Wasser. Die Stadt ließ Bäume fällen, um Unfällen bei schweren Stürmen vorzubeugen. Früher froren die Kanäle im Winter zu und die Anwohner*innen liefen auf der gefrorenen Oberfläche Schlittschuh, was heute nicht mehr möglich ist. Raina kann die drohende Angst nicht ignorieren, dass die Welt, wie sie sie kennt, sich verändern wird. Ihr Heranwachsen ist von Traurigkeit und Angst geprägt. In der Hoffnung, Erwachsene zum Handeln bewegen zu können, hat sie sich mit ihren Freund*innen der Fridays-for-Future-Bewegung angeschlossen.
Abb. 1 Von einem klimaengagierten Kindergartenkind gemaltes Protestplakat. Globaler Klimastreik, Berlin, März 2019. (Quelle: Tanu Biswas) 
Wie viele junge Akivist*innen animiert Raina auch ihre Familie dazu, ihren Lebensstil tatkräftig zu verändern. Ihr Traum davon, eines Tages selbst eine Familie zu gründen, verdüstert sich, da sie unsicher ist, ob sie ihre eigenen Kinder den erwartbaren Umweltbedingungen aussetzen will. Für junge Menschen wie Raina offenbart die Erfahrung, im städtischen Raum mit dem drohenden Schrecken der Klimakrise heranzuwachsen, eine kulturelle Misopädie des modernen Staates und der modernen Familie. Kinder sowie intergenerationelle soziopolitische Interessen sind hier kein zentraler Wegweiser, wenn es etwa darum geht, das schwierige Gleichgewicht zwischen „Wirtschaftswachstum“ und „ökologischer Nachhaltigkeit“ auszutarieren. Vielmehr wird das chronologische Alter hier zu einer maßgeblichen Achse der systemischen Diskriminierung.

Betrachten wir die moderne Kindheit innerhalb der Dreieckskonstellation aus Staat, Familie und Kind. Diese Konstellation ermöglicht es, Kinder als eigenständige Objekte gesellschaftlicher Verwaltung und Kontrolle zu identifizieren. Kinder als „Individuen“ anzusehen, erlaubt zudem eine formale Trennung von ihrem sozialen Hintergrund. Auf den europäischen Kontext bezogen versteht Näsman (1994) dies als „bürokratische Individuierung“. Individuierung bezeichnet dabei die systematische Tendenz, den einzelnen Menschen als Grundeinheit bürokratischer Bearbeitung zu behandeln, Individualisierung hingegen bezieht sich auf das Individuum als psychologische Persönlichkeit (Frønes 1994: 147). Individuierung wird ermöglicht durch ein ausgeklügeltes Klassifizierungs- und Differenzierungssystem zum Zwecke der bürokratischen Versicherung. Dabei wird durch die Gleichbehandlung aller Mitglieder einer Kategorie der Gerechtigkeit Genüge getan. Die Einzigartigkeit jedes Mitglieds einer bestimmten Kategorie hervorzuheben, ist dabei jedoch nicht beabsichtigt (ebd.). Das chronologische Alter ist ein zentraler Faktor, anhand dessen Lebensabschnitte institutionell organisiert werden, vom Kindergarten über Schule, Universität und Arbeitsplatz bis zum Seniorenheim. Dies beinhaltet auch die räumliche und zeitliche Positionierung im Rahmen einer bestimmten wirtschaftlichen Produktionsweise.

Die obligatorischen Aufgaben von Kindern sind systemimmanent, das heißt sie entsprechen immer der jeweils vorherrschenden Produktionsweise (vgl. Qvortrup 2001). Die Art und Weise, auf die Zeit von der Erwachsenengesellschaft gestaltet und beansprucht wird sowie das Maß, in dem Kinder Anspruch auf gesellschaftliche Ressourcen erheben dürfen, werden ebenfalls durch die vorherrschende Produktionsweise beeinflusst (vgl. Qvortrup 2008). Die moderne Ökonomie könnte nicht überleben und gedeihen, würde die schulische Arbeit von Kindern heute nicht einen quantitativ überwiegenden Teil der Kindheit ausmachen (Qvortrup 2001: 97). Eine hohe Einstufung im Human Development Index korreliert heutzutage signifikant mit einer sozialtechnisch hergestellten, existenziellen, räumlich-zeitlichen Positionierung menschlichen Lebens, wie sie für die Moderne spezifisch ist. Wards Appell folgend könnte man von der Annahme ausgehen, dass modernes Humankapital und moderne Kultur vor allem über hochgradig individuierte, individualisierte und institutionalisierte altersbasierte Segregationssysteme reproduziert werden. Derartige Systeme geben auf maßgebliche Weise vor, wie Kindheitserfahrungen global zu standardisieren sind. Damit wird es möglich, weltweit von „[durch]gestalteten Kindheiten“(designed childhoods) 
zu sprechen (vgl. Gutman/de Coninck-Smith 2008). Gillis legt dar, wie die allgegenwärtig angewandte altersbasierte Segregation kinderfeindliche Formen einer altersgruppenspezifischen kulturellen Inselbildung hervorbringt:

„Das moderne Leben ist reich an kindzentrierten Momenten - Weihnachten, Geburtstage, Sommerferien - ausgefeilten Ritualen, von Erwachsenen geschaffen, um Bindungen zu Kindern zu pflegen und sich durch den Zugang zu den eigenen Kindheitserinnerungen selbst zu vergewissern. [...] Die Verinselung von Kindern ist als Schöpfung der Erwachsenen anzusehen, als Reaktion auf ihre eigenen Bedürfnisse und nicht auf die der Kinder. [...] Erwachsene haben Kinder nicht nur physisch verinselt, sondern auch mythische Landschaften erschaffen, die Kindheit in ihren idealisierten Formen aufrechterhalten, auch wenn sie in der realen Welt nicht aufrechterhalten werden kann. [...] Die mythische Geographie besteht aus den mentalen Landkarten, an denen wir uns in einer Welt orientieren, in der physische Orientierungspunkte und Wegweiser oft verborgen oder gar nicht vorhanden sind. Die mythischen Landschaften der Kindheit stellen eine Art Paralleluniversum dar, das Ähnlichkeit mit der physischen Geographie aufweist, aber den Vorzug hat, gegen zeitliche und räumliche Veränderungen gefeit zu sein, die die reale Welt fortwährend transformieren.“ (Gillis 2008: 317; Übersetzung von Andrea Tönjes)

In hoch industrialisierten Gesellschaften scheint der vorherrschende ultimative existenzielle Zweck intergenerationeller Beziehungen in der Förderung individueller Autonomie und Selbstbestimmung zu bestehen, aber nicht in der Förderung von Gemeinschaft (vgl. Abebe/Biswas 2021; Nandy 1984). Die hochgradige Institutionalisierung und Standardisierung menschlicher Lebensläufe, die bereits vor der Geburt einsetzt, ist eine der allgemeinen gesellschaftlichen Operationen zur Umsetzung dieser Werthaltung. Kindheit ist gekennzeichnet durch frühe Jahre von totaler Abhängigkeit, Sicherheit, Unschuld, ausgiebigen Spielens und verpflichtendem Schulbesuch (Honeyman 2013: 167). Die vorrangige Lebensweise eines Kindes besteht darin, sozialisiert zu werden, indem es eine beträchtliche Zeitspanne in der Schüler*innenrolle verbringt. Der Schulbesuch eröffnet dem Kind Zugang zur formalen Bildung, die es benötigt, um sich später in den Erwachsenenmarkt integrieren zu können. Er strukturiert den zeitlichen Übergang vom Kinderzum Erwachsenenstatus, während er zugleich das Kind dabei einschränkt, wie es seine Zeit verbringen kann (James/Jenks/Prout 1998: 41). Eine derartige strukturelle Verortung bietet eine einzigartige Möglichkeit zur Verwaltung und Kontrolle von Kinderpopulationen (ebd.). Die tägliche Zeiteinteilung durch Unterricht, Pausenzeiten und so weiter ist der Schlüssel beim Ordnen des zeitlichen Lebensverlaufs des Kindes durch das moderne Schulwesen (Oswell 2013: 121). Dieses restriktive Ordnen setzt auf der Alltagsebene voraus, dass man lernt, durch den Rhythmus externer Uhren und Kalender ein Gefühl für die Zeit zu entwickeln. Als Teil der modernen Geschichte einer überhitzten Beschleunigung und einer allumfassenden Standardisierung von Zeit (Eriksen 2016; Eriksen 2001) wird Kindheit zu einem hochgradig nach einem Zeitplan durchgetakteten Ereignis (Ennew 1994; Beauvais 2018). Zeit und Geld sind in der Industriegesellschaft aufs Engste miteinander 
verknüpft. Die Uhr dient dabei dazu, synchronisierte Effizienz in immer größerem Maßstab zu fördern (Eriksen 2001: 53; Ennew 1994).

Kindheit ist folglich angefüllt mit durchorganisierten Aktivitäten, die in einem hochgradig instrumentellen Bildungssektor stattfinden.

„Ungeachtet dessen, dass der erlernte Umgang des Kindes mit dem tagesperiodischen Rhythmus biologischer Natur ist, gibt die kulturelle Ordnung der Schlaf- und Wachzeiten (die in modernen Gesellschaften zunehmend den Rhythmen der Arbeitswelt, des Transport-, Kinderbetreuungs- und des Rundfunksystems gehorcht) weitere sozial ,reale Rhythmen vor.“ (Ennew 1994: 127)

Der Spielraum des Kindes für eine selbstbestimmte Erkundung außerhalb der sozial „realen“ Rhythmen wird systematisch eingeschränkt. Leider verliert das erwachsene Gegenüber in seiner Funktion als „Sozialisationsinstanz“ ebenfalls den Zugang zur Erfahrung von Zeitlichkeiten jenseits des singulären Einheitsrhythmus. Aus einer intergenerationellen Perspektive betrachtet offenbart sich hier die Gegenseitigkeit der Misopädie. Mit anderen Worten: Die „unvernünftigen“ Kinder werden darauf reduziert, diejenigen zu sein, die einen spezifischen zeitlichen Rhythmus (noch) lernen müssen. Die „vernünftigen“ Erwachsenen werden entsprechend darauf reduziert, dass sie diesen lehren müssen.

Als Kind ständig als „Schüler*in“ positioniert zu werden, lässt wenig Raum zur Erkundung verschiedener möglicher Dimensionen dessen, wie man mit der Umwelt in Beziehung treten kann. Folglich darf man sich von den „kleinen Entwicklungsinseln der Kindheit“ hin zu den „großen entwickelten Ländern des Erwachsenendaseins" bewegen, solange man auf allen Wegen von Erwachsenen begleitet wird, die sicherstellen, dass das Kind innerhalb der Grenzen bleibt, die seiner jeweiligen Altersgruppe zugewiesen sind. Die metaphorische Reise von „Insel zu Insel“ lässt sich insofern als Ermöglichung systematischer Unfreiheit, Marginalisierung und Unterdrückung begreifen, als das kindliche Alltagsleben zunehmend den Rhythmen der erwachsenenzentrierten Märkte, Transportsysteme, Kinderbetreuungssysteme, Bildungssysteme etc. folgt.

Misopädie zu erkennen bedeutet auch zu sehen, dass Kinder und Kindheit nicht Unfreiheit, Marginalisierung und Unterdrückung ausgesetzt werden können, ohne dass Erwachsene und das Erwachsenendasein dem ebenfalls ausgesetzt sind. Erwachsenenzentrierte Strukturen konstruieren nicht nur Kindheiten, die ständiger Begleitung, Überwachung und Beaufsichtigung bedürfen, sie erfordern auch Erwachsene, die andauernd begleiten, überwachen und beaufsichtigen. Ironischerweise scheint das teleologische intergenerationelle Ziel von „Autonomie“ und „Selbstbestimmung“ durch eine erwachsenenzentrierte Konstruktion weder für Kinder, noch für Erwachsene realisierbar zu sein. Wie auch Colin Ward zeigt, führt die industrielle Urbanisierung zu einer unfreiheitlichen Beziehung zwischen Kindern und ihrer Umwelt. Angesichts der Klimakrise sehen wir, dass diese einschränkende Beziehung für jüngere Generationen belastende Dimensionen annimmt. Die unfreiheitliche Beziehung zur eigenen Umwelt gilt für Erwachsene in ihrer Funktion als „Sozialisationsinstanzen“ ebenso wie für die Kinder, die sozialisiert werden sollen. 
Sich genauer mit Misopädie auseinanderzusetzen, kann eine Möglichkeit sein, der menschlichen Altersvielfalt Rechnung zu tragen. Wards Arbeit sollte nicht allein als Thematisierung der Unfreiheit von Kindern in ihren städtischen Umgebungen verstanden werden. Vielmehr kann man sie als Entschleierung einer unerkannten Dimension der Unfreiheit aller Menschen - egal welchen Alters - gegenüber ihrer Umwelt lesen. Autor*innen wie Colin Ward leisten insofern einen wichtigen Beitrag, als sie dafür sorgen, dass wir nicht vergessen, dass Kinder und Kindheit Teil einer umfassenderen MenschUmwelt-Beziehung sind. Es geht nicht um körperliche Größe, sondern um die Tiefe der Wahrnehmung, um die Freiheit zur Neugier auf das „da draußen“ - wo vielleicht die Horizonte einer freien Beziehung zur (eigenen) Umwelt sanft glitzern. Alberto Caeiro, eines der Heteronyme Fernando Pessoas, beschreibt es so:

„Von meinem Dorf aus sehe ich, was man auf Erden vom Weltall sehen kann...

Darum ist mein Dorf auch so groß wie irgendein anderes Land,

Denn ich bin so groß wie das, was ich sehe,

Und nicht so groß wie ich bin...

In den Städten ist das Leben kleiner

Als hier in meinem Haus auf dem Hügel.

In der Stadt versperren die großen Häuser die Aussicht,

Verdecken den Horizont, stoßen unseren Blick weit weg vom Himmel.

Machen uns klein, denn sie nehmen uns, was unsere Augen uns geben können,

Und machen uns arm, denn unser einziger Reichtum ist Sehen.“

(Pessoa 2015: 37)

\begin{abstract}
Abschließend hoffe ich, Wards Leser*innen ein Fenster geöffnet zu haben, damit sie die enormen kinderfeindlichen Dimensionen der Unfreiheit erkennen und verstehen können, die jedes Urbanisierungsprojekt durchdringen. Altersbasierte Segregationssysteme, wie beispielsweise moderne Schulen, sind unverzichtbare Instanzen jeglicher (modernen) Urbanisierung, um diese Dimensionen der Unfreiheit zu ermöglichen. Die Misopädie der Urbanisierung führt, wie ich dargelegt habe, zu unfreien Beziehungen aller Menschen zu ihrer Umwelt - ganz unabhängig vom Alter. Wollte man sich einer Fortführung von Wards Arbeit widmen, könnte solch ein Bemühen beim Erkennen und Verstehen von Misopädie ansetzen.
\end{abstract}

Übersetzung aus dem Englischen von Andrea Tönjes für SocioTrans - Social Science Translation \& Editing Services.

Dieser Artikel wurde durch den Open-Access-Publikationsfonds der Universität Bayreuth gefördert. 


\section{Autor_innen}

Tanu Biswas ist eine interdisziplinäre Bildungsphilosophin. Sie forscht an der Schnittstelle von Childism, Kindheitsforschung, Pädagogik, intergenerationeller Klimagerechtigkeit und Dekolonialität.

biswas.tanushree@gmail.com

\section{Literatur}

Abebe, Tatek / Biswas, Tanu (2021): Rights in education: Outlines for a decolonial, childist reimagination of the future. Commentary to Ansell and colleagues. In: Fennia. International Journal of Geography 199/1, 118-128.

Beauvais, Clémentine (2018): Thinking the adult-child relationship with existentialism. In: Spyros Spyrou / Rachel Rosen / Daniel Thomas Cook (Hg.), Reimagining childhood studies. London: Bloomsbury Academic, 57-74.

Bonnardel, Yves (2015): La domination adulte: L'oppression des mineurs. Mereville: Myriadis.

Earth Justice (2019): Communication to the committee on the rights of the child. In the case of Chiara Sacchi (Argentina); Catarina Lorenzo (Brazil); Iris Duquesne (France); Raina Ivanova (Germany); Ridhima Pandey (India); David Ackley, III, Ranton Anjain, and Litokne Kabua (Marshall Islands); Deborah Adegbile (Nigeria); Carlos Manuel (Palau); Ayakha Melithafa (South Africa); Greta Thunberg and Ellen-Anne (Sweden); Raslen Jbeili (Tunisia); Carl Smith and Alexandria Villaseñor (USA); Petitioners, V. Argentina, Brazil, France, Germany \& Turkey. https://earthjustice.org/sites/default/files/files/2019.09.23crc-communication-sacchi-et-al-v.-argentina-et-al-redacted.pdf (letzter Zugriff am 12.12.2020).

Ennew, Judith (1994): Time for children or time for adults. In: Jens Qvortrup / Marjatta Bardy / Giovanni Sgritta / Helmut Wintersberger (Hg.), Childhood matters. Social theory, practice and politics. Aldershot: Avebury, 125-143.

Eriksen, Thomas Hylland (2001): Tyranny of the moment - fast and slow. Time in the information age. London/Sterling: Pluto Press.

Eriksen, Thomas Hylland (2016): Overheating. An anthropology of accelerated change. London/Sterling: Pluto Press.

Frønes, Ivar (1994): Dimensions of childhood. In: Jens Qvortrup / Marjatta Bardy / Giovanni Sgritta / Helmut Wintersberger (Hg.), Childhood matters. Social theory, practice and politics. Aldershot: Avebury, 145-164.

Gill, Tim (2021): Urban playground: How child-friendly planning and design can save cities. London: Routledge.

Gillis, John R. (2008): The Islanding children. Reshaping the mythical landscapes of childhood. In: Marta Gutman / Ning de Coninck-Smith (Hg.), Designing modern childhoods. History, space, and the material culture of children. Piscataway: Rutgers University Press, 316-330.

Gutman, Marta / de Coninck-Smith, Ning (Hg.) (2008): Designing modern childhoods. History, space, and the material culture of children. Piscataway: Rutgers University Press.

Honeyman, Susan (2013): Trans(cending) gender through childhood. In: Anna Mae Duane (Hg.), The children's table: Childhood studies and the humanities. Athens: University of Georgia Press, 167-179.

James, Allison / Jenks, Chris / Prout, Alan (1998): Theorizing childhood. Cambridge: Polity Press.

Liebel, Manfred (2020): Unerhört: Kinder und Macht. Weinheim: Beltz Juventa.

Nandy, Ashis (1984): Reconstructing childhood. A critique of the ideology of adulthood. In: Alternatives 10/3, 359-375.

Näsman, Elisabet (1994): Individualization and institutionalization of childhood in today's Europe. In: Jens Qvortrup / Marjatta Bardy / Giovanni Sgritta / Helmut Wintersberger (Hg.), Childhood matters. Social theory, practice and politics. Aldershot: Avebury, 165-187. 
Oswell, David (2013): The agency of children. From family to global human rights. New York: Cambridge University Press.

Pessoa, Fernando (2015): Wenn das Herz denken könnte. Sätze aus dem Gesamtwerk. Frankfurt am Main: Fischer.

Qvortrup, Jens (2001): School-work, paid work and the changing obligations of childhood. In: Angela Bolton / Phillip Mizen / Christopher Pole (Hg.), Hidden hands. International perspectives on children's work and labour. London: Routledge, 91-108.

Qvortrup, Jens (2008): Childhood in the welfare state. In: Allison James / Adrian L. James (Hg.), European childhoods. Cultures, politics and childhoods in Europe. Basingstoke: Palgrave Macmillan, 216-232.

Rollo, Toby (2018): Feral children. Settler colonialism, progress, and the figure of the child. In: Settler Colonial Studies 8/1, 60-79.

Ward, Colin (1978): Das Kind in der Stadt. Frankfurt am Main: Fischer. 